\title{
Assessment of Resection Margins in Bone Tumor Surgery
}

\author{
Corentin Malherbe $\mathbb{D}^{1},{ }^{1}$ Bernard Crutzen $\mathbb{D}^{2},{ }^{2}$ Jean Schrooyen $\mathbb{D},{ }^{1}$ Giovanni Caruso $\mathbb{D}$, \\ Frédéric Lecouvet $\mathbb{D}^{2},{ }^{2}$ Christine Detrembleur $\mathbb{D}^{3},{ }^{3}$ Thomas Schubert $\mathbb{D}^{1},{ }^{1,3}$ \\ and Pierre-Louis Docquier (iD)
}

\author{
${ }^{1}$ Service de Chirurgie Orthopédique et Traumatologique, Cliniques Universitaires Saint-Luc, Avenue Hippocrate 10, \\ 1200 Brussels, Belgium \\ ${ }^{2}$ Département D'Imagerie Médicale, Cliniques Universitaires Saint-Luc, Avenue Hippocrate 10, 1200 Brussels, Belgium \\ ${ }^{3}$ Neuro Musculo Skeletal Lab (NMSK), Institut de Recherche Expérimentale et Clinique, Université Catholique de Louvain, \\ Secteur des Sciences de la Santé, Avenue Mounier 53, B-1200 Brussels, Belgium \\ Correspondence should be addressed to Pierre-Louis Docquier; pierre-louis.docquier@uclouvain.be
}

Received 11 April 2020; Revised 24 August 2020; Accepted 27 November 2020; Published 10 December 2020

Academic Editor: Manish Agarwal

Copyright @ 2020 Corentin Malherbe et al. This is an open access article distributed under the Creative Commons Attribution License, which permits unrestricted use, distribution, and reproduction in any medium, provided the original work is properly cited.

\begin{abstract}
Limb salvage surgery is now the preferred procedure for bone tumor surgery. To decrease the risk of local recurrence, it is crucial to obtain adequate resection margins. The obtained margins must be evaluated postoperatively because they influence what treatment is given subsequently when margins are not adequate (e.g., surgical revision and radiotherapy). The study aims to evaluate margin assessment of tumor specimen by MRI compared to conventional histology (to establish the viability of using MRI) and assess the accuracy of a patient-specific instrument when narrow margins were aimed. The resection margins in 12 consecutive patients that were operated on for bone tumor resection were prospectively analyzed using three methods: MRI of the resection specimen, macroscopic evaluation of specimen slices, and microscopic pathological evaluation. The assessments were qualitative (R0, R1, and R2) and quantitative (distance in mm). MRI, macroscopic, and microscopic margins generated similar results for both the qualitative (all resections were R0) and quantitative assessments. The median error in safe margins was $2 \mathrm{~mm}$ with a surgical guide (PSI) and $5 \mathrm{~mm}$ without a surgical guide. Local recurrences were not detected after a mean follow-up period of 3.7 years (range, 2.1-5 years); however, four patients died during the study. In conclusion, MRI is a valuable tool for assessing safe margins. When specimens are not available for pathological assessment (e.g., extracorporeally irradiated autograft or autoclaved autograft), MRI could be used to evaluate margins. In particular, when tumor volume is high, MRI could also help to focus the pathological examination on areas of concern.
\end{abstract}

\section{Introduction}

When possible, limb salvage is the preferred procedure to manage malignant bone tumors. In such cases, "en bloc" surgical resection is required without penetrating the tumor [1-6]. During the procedure, the surgeon resects the tumor while retaining a safe continuous margin of healthy tissues around it. Precise preoperative planning is required to respect margins (without penetrating the tumor) and to avoid cutting neurovascular structures. No universal quantitative recommendation is available on optimal margin size in the published literature. [7] The qualitative recommendation for margins is $\mathrm{R} 0$ resection, [8] which is when no residual tumor remains in the patient. R1 (removal of all macroscopic disease, but microscopic margins are positive for tumor) and R2 (gross residual tumor that was not resected) resections are clearly linked to a worse prognosis; [9] consequently, a second surgery or adjuvant radiotherapy is necessary [10].

Currently, the pathological examination of bone tumor takes time for voluminous tumors. The time necessary to decalcify, embed in paraffin, and analyze the specimen is ten to fifty days. Furthermore, obtaining an accurate analysis of the whole specimen is often difficult. The postoperative 
evaluation of resection margins by MRI [11-13] has been proposed in the published literature. MRI evaluation is more rapid and can be used to assess the whole specimen easily, including those with voluminous tumors. The study objectives are as follows:

(1) To assess the value of an MRI and its accuracy in assessing the margins of the resection specimen

(2) To assess the accuracy of a patient-specific instrument (PSI) in obtaining the planned margin

\section{Materials and Methods}

2.1. Ethics Committee Agreement. Agreement of the central ethics committee was obtained and was given the following number: UCL/MGS/001 (2009/02 AVR/126).

2.2. Patient Series. Data were obtained prospectively between January 2015 and January 2017. Based on our protocol, inclusion criteria were primary bone sarcoma accessible to resection surgery. There were five osteosarcomas, six Ewing's sarcomas, and one fibrous dysplasia. Diagnosis of fibrous dysplasia was only possible postoperatively, as imaging was unclear; thus, the decision to perform resection was made without a previous biopsy. Localizations were two iliac bones, six femurs, one ulna, two radiuses, and one fibula. Three patients were already metastatic at the time of tumor discovery. The time between surgery and the last follow-up was 3.7 years (range: $2.1-5$ years). Four patients died in the two postoperative years (Table 1).

2.3. Preoperative MRIEvaluation. Two preoperative MRIs of the bone tumor were obtained in all cases (with general anesthesia for children): prechemotherapy MRI (within one month before the first course of chemotherapy) and postchemotherapy MRI (at the end of chemotherapy and before surgery). A Siemens MAGNETOM Verio 3 Tesla MRI was used, and the following specific sequences were used for the preoperative exams: axial $3 \mathrm{D} \mathrm{T} 1$ - and $3 \mathrm{D}$ T2-weighted images, coronal proton density-weighted images with fat saturation, and axial and sagittal T1-weighted images with gadolinium enhancement were obtained. MRI acquisition parameters were specified as follows: reconstruction matrix $176 \times 176,0.5 \mathrm{~mm}$ section thickness and $0.5 \mathrm{~mm}$ spacing between slices. The MRIs were reconstructed by multiplanar reconstruction (MPR) and were saved in DICOM format with a picture archiving and communication system (PACS; Carestream Health, NY, USA).

2.4. Surgery. Surgery was executed with PSI in seven cases and without PSI in five cases. In seven cases, a $1 \mathrm{~cm}$ margin was planned to preserve a joint or an epiphysis, and a PSI was used (to increase the accuracy of the resection margins $[14,15])$. PSI was also used in one case where a step-cut was planned to increase stability and for allograft cutting in another patient.

When the planned margin exceeded $2 \mathrm{~cm}$, PSI was not used. This was the case, for example, when a minimal $17 \mathrm{~cm}$ resection was needed to implant a growth prosthesis. The PSI was designed by an engineer (3D-Side $\left.{ }^{\circledR}\right)$. The tumor was delineated on MRI using the software developed by 3DSide ${ }^{\circledR}$. The preferred MRI used to delineate the tumor type was T1 [16]. The CT scanner of the preoperative PET scanner was used with minimal $1 \mathrm{~mm}$ section thickness. Tumor volume obtained from MRI was merged with the CT scanner to create a $3 \mathrm{D}$ scan in which the tumor was visible (by using the software developed by 3D-Side). The PSI was created from the 3D scanner based on the margins selected by the surgeon. After tumor resection, the specimen was oriented using surgical threads (craniocaudal). A sample was collected from the tumor for genetic analysis, and the tumor was immersed in $4 \%$ formalin.

2.5. Postoperative MRI Evaluation of the Resection Specimen. MRIs of the resected tumor specimen were obtained using a Philips Achieva 3 Tesla MRI. The specific sequences were axial 3D T1- and 3D T2-weighted MRI. The MRI was performed within $6 \mathrm{~h}$ of surgery. The same MRI machine was used for the entire series. Specimens were oriented according to the long axis of the long bone or the craniocaudal axis of the pelvic bone. MRI acquisition parameters were specified as follows: reconstruction matrix $176 \times 176,0.5 \mathrm{~mm}$ section thickness, and $0.5 \mathrm{~mm}$ spacing between slices. The MRIs were reconstructed by MPR and were saved in DICOM format with PACS (Carestream Health, NY, USA). Through MPR, MRI slices were created similar to the macroscopic cuts. MRIs were analyzed, classified, and measured by three independent observers (i.e., blindly) based on the standardized classification of the Union for International Cancer Control (UICC) [17]. Observers had access to the preresection MRI to help analyze the specimen. The three observers included an experienced radiologist used to tumor imaging, a junior radiologist with no experience in bone tumor surgery, and an experienced orthopedic surgeon used to tumor surgery. UICC classification distinguished R0 with adequate safe margins ( $>1 \mathrm{~mm}), \mathrm{R} 1$ as possible microscopic residuals (minimal margin between 0 and $1 \mathrm{~mm}$ ), and $\mathrm{R} 2$ as macroscopic residual disease $(<0 \mathrm{~mm})$. Examiners received a folder with schematic oriented representations of the margins and axes to use for making measurements (Figure 1). Measurements were requested for each saved MRI cut (T1 and T2) (equivalent to the macroscopic cuts) (Figures 2 and 3) and for the whole MRI.

2.6. Macroscopic Evaluation. Within the hour following MRI (i.e., before cutting), the specimen was inked. The specimen was sliced in a coronal plane using a band saw. Each slice was oriented and numbered in ascending number from posterior to anterior. A picture of each section was captured with a graduated scale in the same layout (Figure 4). The size and margins of the specimen were measured by an orthopedic resident and were expressed in millimeters. The specimens were subsequently used for macroscopic analysis by both the pathologist and orthopedic resident 
Table 1: Patients' data.

\begin{tabular}{|c|c|c|c|c|c|c|c|}
\hline$N$ & Age (years) ${ }^{*}$ & Gender & Tumor location & Type & Surgical guide (PSI) & Presence of metastases & Evolution \\
\hline 1 & 11 & M & Femur & OS & Yes & No & NED \\
\hline 2 & 11 & $\mathrm{~F}$ & Pelvis & EwS & Yes & No & NED \\
\hline 3 & 21 & $\mathrm{~F}$ & Femur & $\mathrm{FD}$ & Yes & No & NED \\
\hline 4 & 9 & $\mathrm{~F}$ & Radius & OS & Yes & No & NED \\
\hline 5 & 7 & $\mathrm{~F}$ & Radius & EwS & Yes & No & NED \\
\hline 6 & 6 & $\mathrm{~F}$ & Femur & EwS & No & No & NED \\
\hline 7 & 12 & M & Femur & OS & No & Yes & Deceased \\
\hline 8 & 9 & M & Femur & OS & No & No & NED \\
\hline 9 & 11 & M & Femur & OS & No & No & NED \\
\hline 10 & 36 & M & Ulna & EwS & No & Yes & Deceased \\
\hline 11 & 14 & M & Pelvis & EwS & Yes & No & Deceased \\
\hline 12 & 17 & $\mathrm{M}$ & Fibula & EwS & Yes & Yes & Deceased \\
\hline
\end{tabular}

M: male; F: female; NED: no evidence of disease; OS: osteosarcoma; EwS: Ewing's sarcoma; FD: fibrous dysplasia. ${ }^{*}$ At the time of surgery.

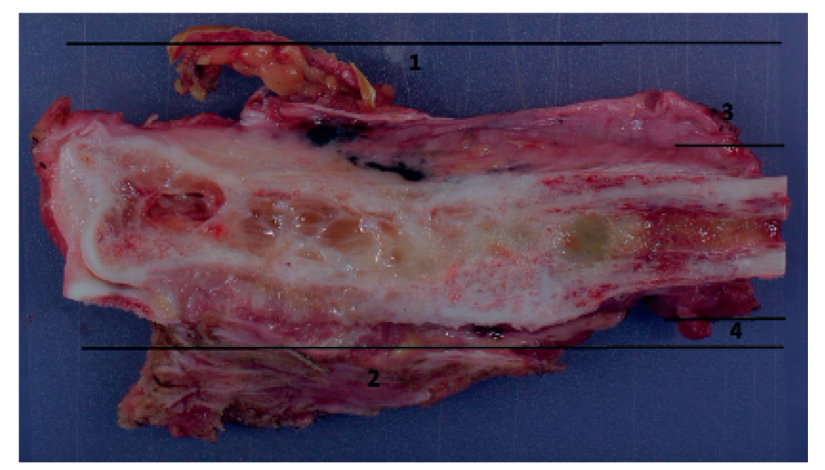

FiguRE 1: A 36-year-old patient with Ewing's sarcoma of the distal ulna. Example of schematic oriented representation of margins to be measured. The corresponding MRI slices (created by MPR) were given for measures.

based on UICC classification. After macroscopic analysis, the specimen was immersed in $4 \%$ formalin.

2.7. Pathological Evaluation. Determination of surgical margins by pathologists is now standardized $[18,19]$. A second macroscopic evaluation of the tumor margin was performed by the pathologist to locate areas with the narrowest margins. Tissue samples were taken at these locations for histology. These blocs were embedded in paraffin and cut to a thickness of 5 microns before staining with Hematoxylin and Eosin. The distance between the inked border and tumor was measured microscopically. The measure was expressed in millimeters. The pathologist again evaluated the quality of the resection based on the UICC classification, using none of the postoperative MRI information. The pathologist rated the type, grade [20], and stage of resection.

2.8. Statistical Analysis. Macroscopic measurements (which are considered as the gold standard) were compared with the MRI measurements of the three independent observers. All statistical analyses were performed using SigmaPlot software 13.0. A Kruskal-Wallis test (one-way analysis of variance on ranks) was used to evaluate agreement among the observers. We also used the Bland and Altman method and Passing-Bablok regression to compare and calculate the bias of each value obtained by the three observers and macroscopy. Using Passing-Bablok regression, intercept A represented the measure of the systematic differences between values. If the confidence interval for intercept A did not contain that value 0 , it was concluded that $A$ was significantly different from 0 and that both methods differed, at least, by a constant amount. Slope B was the measure of the proportional difference between values. If the confidence interval for slope $B$ did not contain value 1 , it was concluded that $\mathrm{B}$ was significantly different from 1 and that there was at least a proportional difference between the two methods. We checked the correlation between measures using Spearman's correlation. To compare planned safe margins and the actual achieved margins with and without PSI, we evaluated the difference between the four independent measurements and the planned distance (in the operating protocol). We calculated the mean, median, maximum error, and standard deviation. For example, if it was planned to cut the distal femur at $175 \mathrm{~mm}$ from the medial condyle, we checked whether that distance was $175 \mathrm{~mm}$. To compare planned safe 


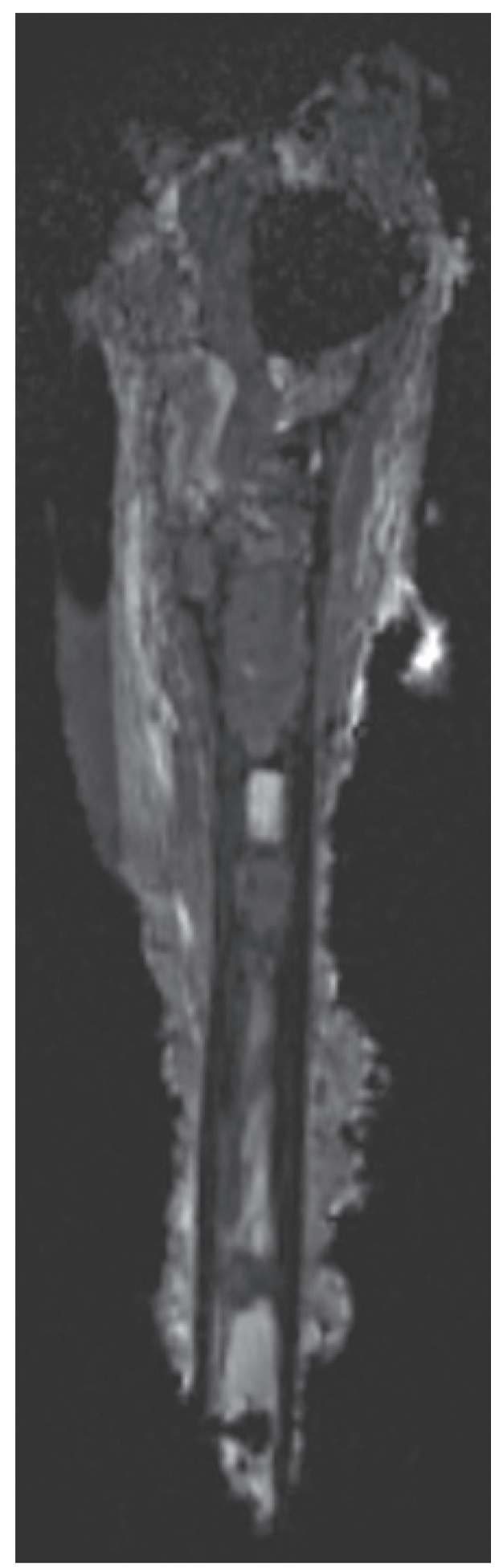

(a)

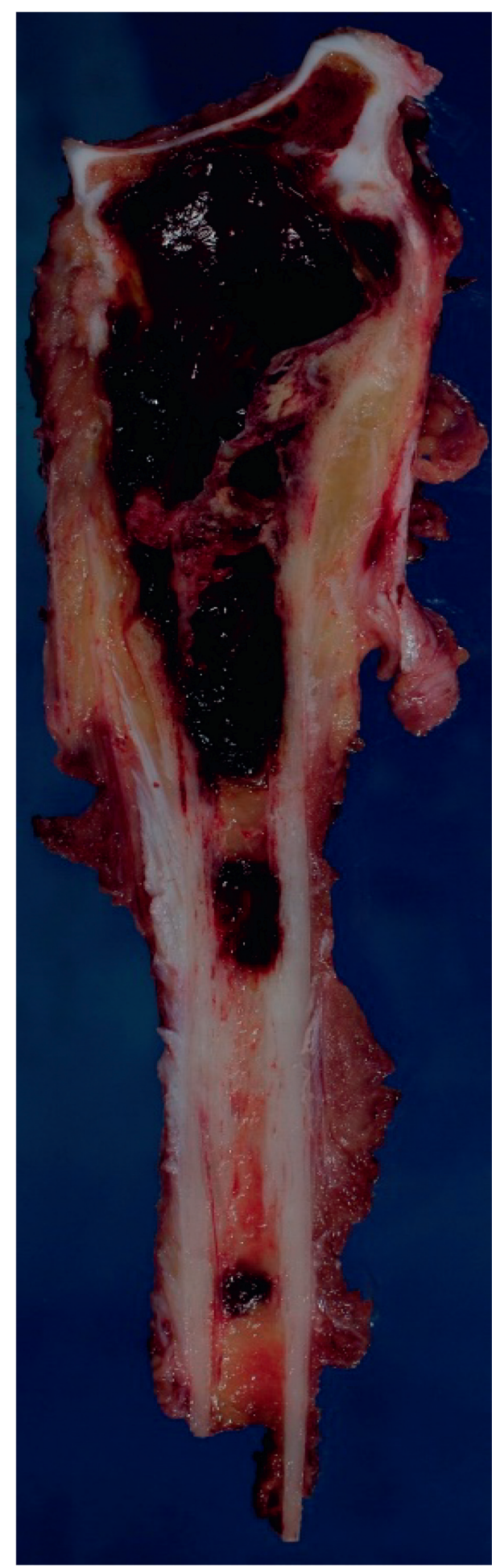

(b)

FIgure 2: Continued. 


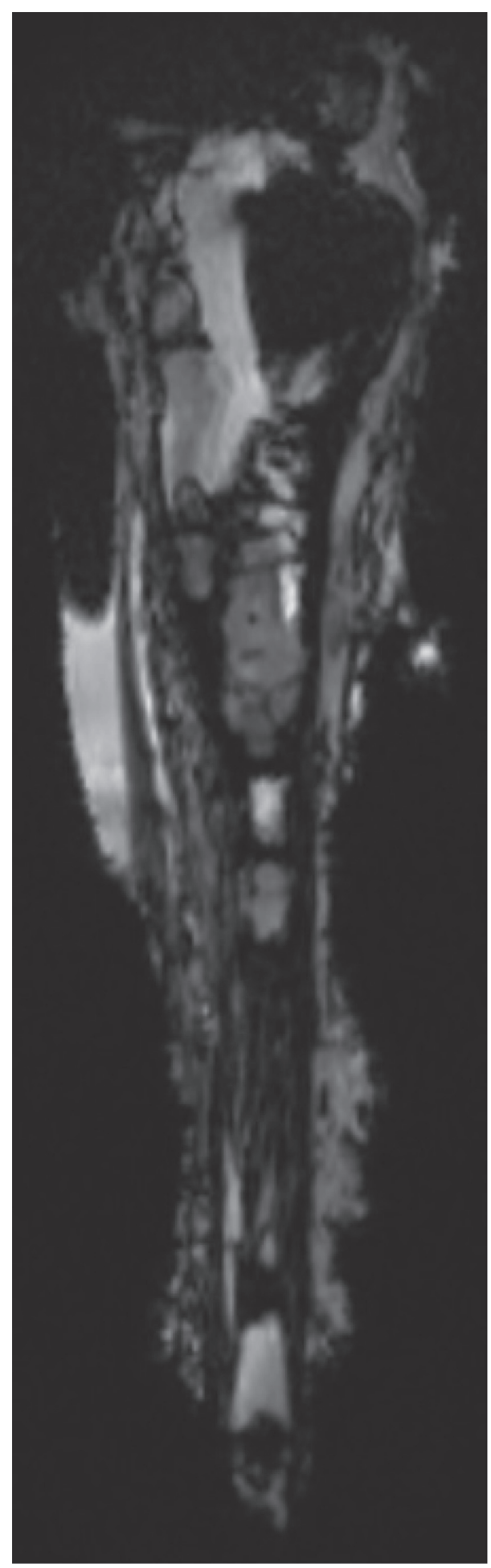

(c)

FiguRe 2: A 9-year-old girl with osteosarcoma of the distal radius. A step-cut was performed proximally for the stability of surgical reconstruction by using a PSI. Two margins were measured (medial and lateral part of the step-cut). Note the difficulty to visualize the cortical bone in the step-cut with MRI. (a) T1-weighted MRI; (b) macroscopic cut; (c) T2-weighted MRI.

margins with PSI, we compared the planned margins with the smallest obtained margins. For example, if a $10 \mathrm{~mm}$ margin was planned and the smallest margin was measured at $8 \mathrm{~mm}$, the error was $2 \mathrm{~mm}$.

\section{Results}

Agreement among MRI, macroscopic, and pathological evaluation was perfect for the qualitative assessment. All 


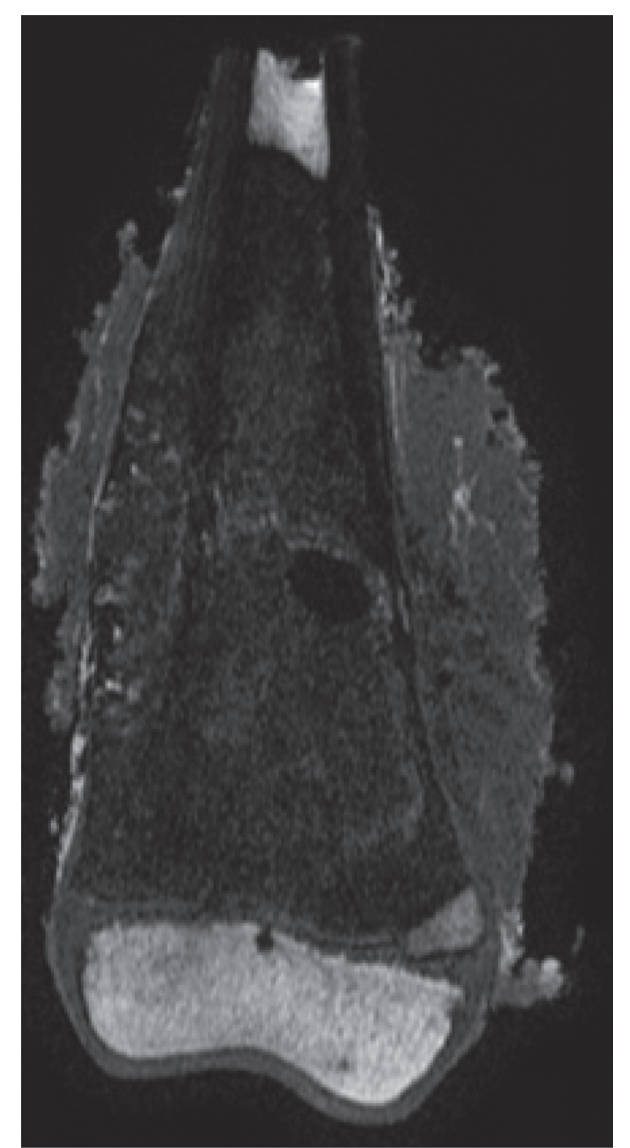

(a)

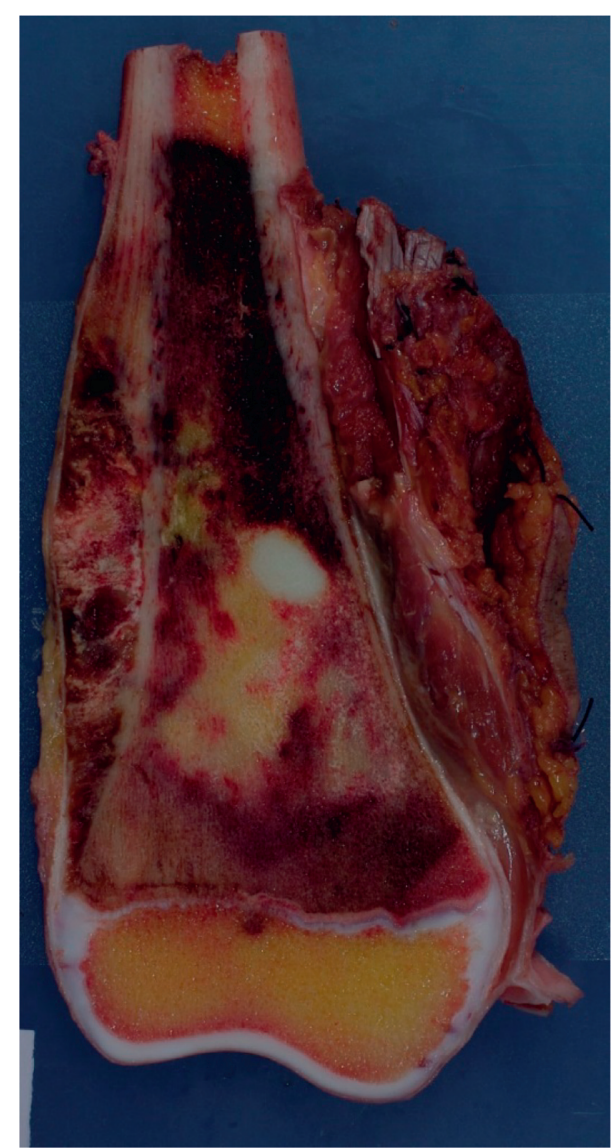

(b)

Figure 3: Continued. 


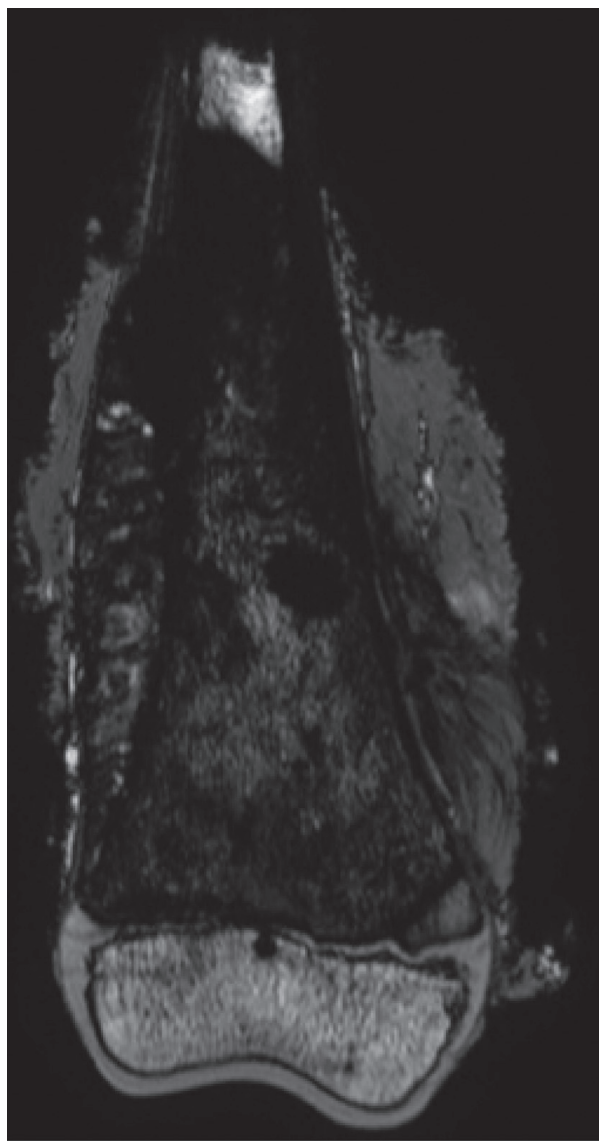

(c)

Figure 3: An 11-year-old boy with distal femur osteosarcoma. No PSI was used because $17.5 \mathrm{~cm}$ bone resection was necessary for a growth prosthesis. One margin was measured. (a) T1-weighted MRI; (b) macroscopic cut; (c) T2-weighted MRI.

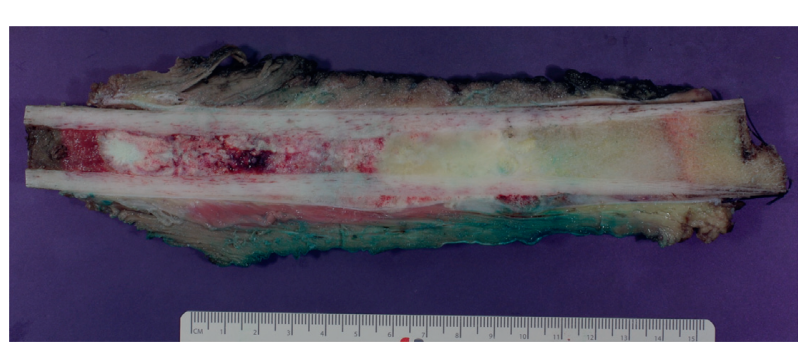

Figure 4: Macroscopy of femoral osteosarcoma with two cuts, one horizontal and one step-cut.

bone resections were evaluated as being R0 using all three methods. There was no statistically significant difference between the MRI evaluation by the three observers (senior radiologist, junior radiologist, and senior orthopedist) versus the macroscopic evaluation (based on 145 measures) $(p=0.94)$ (Table 2).

A very strong correlation was found between the three observers. A very strong correlation was also found between macroscopy and junior radiologist and senior orthopedist (Table 3). A strong correlation was found between the senior radiologist and macroscopy.
If the confidence interval for intercept A did not contain the value 0 , it was concluded that $A$ was significantly different from 0 and both methods differed at least by a constant amount. If the confidence interval for slope B did not contain the value 1 , it was concluded that B was significantly different from 1 and there was at least a proportional difference between the two methods.

The bias between methods was very small; however, Passing-Bablok regression indicated that the methods were not equal (Table 4).

When PSI was not used, the median and maximum margins of error (compared to the planned margin) were $5 \mathrm{~mm}$ and $11 \mathrm{~mm}$, respectively (Table 5).

When using PSI, the median and maximum margins of error were $2 \mathrm{~mm}$ and $6 \mathrm{~mm}$, respectively (Tables 6 and 7). This calculation was made on five patients. One patient had more than the expected margin because the volume of the tumor had reduced by a few centimeters during chemotherapy. For another patient (patient 5), the cut was performed accurately in accordance with the plan and PSI, but the measured margin was $9 \mathrm{~mm}$ less than expected due to a mistake in surgical planning. Fortunately, the margin was still R0. This result was caused by the bad resolution of the 
TABLE 2: Equivalence between MRI and macroscopic evaluation (Kruskal-Wallis one-way analysis of variance on ranks) ( $n=145$ ).

\begin{tabular}{lr}
\hline & Median (range) in mm \\
\hline MRI senior radiologist & $20[14-26.5]$ \\
MRI senior orthopedist & $21[15-29]$ \\
MRI junior radiologist & $20.5[13.8-26]$ \\
Macroscopy & $22[13-26]$ \\
\hline
\end{tabular}

TABle 3: Differences between the methods according to Passing-Bablok regression.

\begin{tabular}{lccc}
\hline & Intercept A [CI] & Slope B [CI] & Correlation coefficient \\
\hline MRI senior radiologist/senior orthopedist & $-1.8[-3$ to 0$]$ & $1.1[1$ to 1.1$]$ & $0.888(p<0.0001)$ \\
MRI senior radiologist/macroscopy & $-2.1[-4.8$ to -0.3$]$ & $1.1[1.0$ to 1.2$]$ & $0.736(p<0.0001)$ \\
MRI senior orthopedist/macroscopy & $-2.9[-4.5$ to -1.4$]$ & $1.1[1.1$ to 1.2$]$ & $0.896(p<0.0001)$ \\
MRI senior radiologist/junior radiologist & $0[-1.1$ to 0$]$ & $1[1.0$ to 1.0$]$ & $0.882(p<0.0001)$ \\
MRI senior orthopedist/junior radiologist & $1.3[0.4$ to 2.1$]$ & $0.9[0.9$ to 0.97$]$ & $0.935(p<0.0001)$ \\
MRI junior radiologist/macroscopy & $-0.8[-1.8$ to 0$]$ & $1.0[1.0$ to 1.1$]$ & $0.901(p<0.0001)$ \\
\hline
\end{tabular}

TABLE 4: Bias between the methods.

\begin{tabular}{lccc}
\hline & Bias $(\mathrm{mm})$ & Standard deviation & Margin bigger for \\
\hline MRI senior radiologist/senior orthopedist & 1.19 & 7.3 & Orthopedist \\
Macroscopy/MRI senior radiologist & -1.37 & 8.5 & Macroscopy \\
MRI senior orthopedist/macroscopy & 0.11 & 5.7 & Macroscopy \\
MRI senior radiologist/junior radiologist & 1.26 & 6.9 & Junior radiologist \\
MRI senior orthopaedist/junior radiologist & 0.16 & 4.15 & Junior radiologist \\
MRI junior radiologist/macroscopy & -0.49 & 4.86 & Junior radiologist \\
\hline
\end{tabular}

TABLE 5: Reliability without PSI (in $\mathrm{mm}$ ).

\begin{tabular}{lccc}
\hline & Median & Max & Std dev \\
\hline MRI senior radiologist & 5 & 8 & 2.6 \\
MRI senior orthopedist & 6 & 11 & 3.7 \\
MRI junior radiologist & 3 & 7 & 2.4 \\
Macroscopy & 8 & 10 & 3.5 \\
ALL & 5 & 11 & 3.0 \\
\hline
\end{tabular}

TABLE 6: Reliability with PSI (in $\mathrm{mm}$ ) with patient 5.

\begin{tabular}{lccc}
\hline & Median & Max & Std dev \\
\hline MRI senior radiologist & -2 & -9 & 3.6 \\
MRI senior orthopedist & -2 & -6 & 2.2 \\
MRI junior radiologist & -2 & -9 & 2.7 \\
Macroscopy & -2 & -9 & 3.9 \\
ALL & -2 & -9 & 3.1 \\
\hline
\end{tabular}

prechemotherapy MRI, inducing an error in the volume planning of the tumor.

\section{Discussion}

Quantitative comparison between MRI and macroscopic evaluations of resection specimens is not available in the published literature. All existing MRI studies compared tumor size before surgery and macroscopically [21-23]. These studies demonstrated a systematic error between the two methods ranging from 5.9 to $19 \mathrm{~mm}$. The current study
TABLE 7: Reliability with PSI (in $\mathrm{mm}$ ) without patient 5.

\begin{tabular}{lccc}
\hline & Median & Max & Std dev \\
\hline MRI senior radiologist & -2 & -3 & 0.8 \\
MRI senior orthopedist & -2 & -6 & 2.0 \\
MRI junior radiologist & -2 & -3 & 0.7 \\
Macroscopy & -2 & -3.5 & 1.3 \\
ALL & -2 & -6 & 1.2 \\
\hline
\end{tabular}

also obtained similar margins of error when comparing MRI to macroscopic assessment. The current prospective study followed the same protocol and the same conditions (the same MRI was used). The MRI and macroscopic measurements were carried out by the three readers, including a junior orthopedist, to reduce human and material bias.

The MRI evaluation was performed in thirty minutes, and the radiologist assessment took no more than fifty minutes. If rapid access to MRI is possible, the full analysis can be done in less than an hour. Therefore, this MRI assessment is more rapid than the classical pathological assessment (paraffin-embedded sections) (10 to 15 days). A frozen section is possible but cannot be used in situations like ECRT where the bone is reimplanted. It also can have a sampling error as the entire margin cannot be assessed.

For MRI, the presence of a sterile plastic wrap did not alter the picture quality of MRI because no artifact was generated by the plastic wrap.

The space between slices was $0.5 \mathrm{~mm}$ when measured using the MRI and 3-4 mm when measured macroscopically 
under the best conditions (slices by band saw). MRI facilitated the use of thinner slices and provided much more information, such as skip metastasis and small lesions. MRI specimens can be compared to preoperative MRIs to determine the location of the lesion more accurately. However, the pathological analysis of the resected specimen provides information that MRI cannot provide, such as the percentage of tumor necrosis. Thus, pathological analysis is required when this information is needed to adjust adjuvant chemotherapy protocols. When tumor specimens are not available for pathology assessment, MRI is the optimal tool for assessing surgical margins, such as the extracorporeal irradiation of specimens, reimplantation, and autoclaved autografts. MRI also complements in-depth pathological analyses of entire tumor specimens, highlighting questionable areas for pathologists.

Some issues were encountered when analyzing the margins with MRI. When the cortical bone is not surrounded by soft tissue, it cannot be distinguished by MRI. Soft tissue tends to retract after the bone is cut and when immersed in formalin (Figure 2). Often, the cortical bone at the site of the section is not surrounded by soft tissue but by air; consequently, it cannot be distinguished easily (black on black in an MRI). The MRI tended to minimalize the margin in comparison to macroscopy. The safe margin tends to be $1.5 \mathrm{~mm}$ less than the planned range due to the thickness of the saw blade. Some bone material is also lost when a bone is cut due to the vibration of the saw blade (called the kerf effect) [24].

The senior radiologist recorded smaller margins compared to the junior radiologist and senior orthopedist (Table 2). This difference could be explained by the method used by the senior radiologist. The senior radiologist interpreted the MRI in its entirety by visualizing the slices before and after they were selected. If the tumor was closer to the margin on an adjacent slice, the senior radiologist measured the margin accordingly. This approach might explain the systematically smaller margin recorded by the senior radiologist. In comparison, the orthopedist and junior radiologist selected slices without evaluating adjacent slices.

When using PSI, the median error was $2 \mathrm{~mm}$ (better accuracy compared to without PSI); however, sufficient planning is required. When planning is inadequate (i.e., the tumor is not appropriately delineated on MRI), there is a systematic effect on the final measurement of the margin. Thus, meticulous analysis of the preoperative MRI is required to delineate the limit of the tumor. If the quality of the preoperative exam is poor, it should be repeated.

The accuracy of MRI in assessing the bone extension of tumors was assessed by Thompson et al. [16]. The authors obtained a difference of $13 \mathrm{~mm}$ for the mean tumor size when comparing MRI to histopathology. We did not obtain this difference in our four measurements. The human factor in surgery is always present. Thus, for various reasons, using a margin that is too narrow is dangerous. In general, the bone margin is planned based on the prechemotherapy MRI, whereas the soft tissue margin is planned based on the postchemotherapy MRI. Some recent studies also suggested planning bone margins based on postchemotherapy
MRI [25]. We can overestimate the bone margin if the tumor shrinks during chemotherapy. To exclude the chance that mistakes in cutting were masked by a decline in tumor size, we also checked the size of the specimens (planned and achieved) when comparing the two results. Tumor volume only declined in one case; thus, this case was excluded from the analysis measuring PSI accuracy. In the series without PSI, we only checked the accuracy of specimen size.

This study has some limitations. We did not compare T1 and T2 measurements. Some studies showed closer margins with T1-weighted measurements. To assess the marginal cortex with greater precision, T1 and T2 are necessary. All of the histologic analyses were performed by a single observer and only once. Only 12 patients were included in the study, of which seven benefited from PSI.

\section{Conclusions}

This study shows that the MRI evaluation of the resection specimen was equivalent to the pathological evaluation, both qualitatively and quantitatively. MRIs are limited in that they cannot inform the pathologist on the extent of necrosis and type of tumor. When this information is needed, MRI cannot replace pathological evaluation. Surgical resection with PSI was more accurate than that without PSI (median error of $2 \mathrm{~mm}$ versus $5 \mathrm{~mm}$ ); however, the series was too small to generate recommendations on margins. Despite PSI, meticulous preoperative planning on MRIs remains mandatory. To preserve a joint or an epiphysis, PSI is recommended to decrease the margin.

\section{Data Availability}

Due to the specific nature of the pathology and the limited number of cases, it is easy to identify patients despite the anonymization of the data. These data will be shared on request from members of the medical profession. The statistical data used to support the findings of this study are available from the corresponding author upon request. The measurement data used to support the findings of this study are available from the corresponding author upon request.

\section{Conflicts of Interest}

The authors declare that they have no conflicts of interest.

\section{Acknowledgments}

Funds were received from the Belgian Foundation against Cancer (Grant FAF-C/2016/748).

\section{Supplementary Materials}

The statistical data and the measurement data are available on a statistic.docx and measurementdata.xls. These files are also available from the corresponding author upon request. (Supplementary Materials) 


\section{References}

[1] L. Xin and V. M. Moretti, A. O. Ashana and R. D. Lackman, Impact of close surgical margin on local recurrence and survival in osteosarcoma," International Orthopaedics, vol. 36, no. 1, pp. 131-137, 2012.

[2] N. Kawaguchi, A. R. Ahmed, S. Matsumoto, J. Manabe, and Y. Matsushita, "The concept of curative margin in surgery for bone and soft tissue sarcoma," Clinical Orthopaedics and Related Research, vol. 419, pp. 165-172, 2004.

[3] V. Manabe, M. A. Smolle, J. Szkandera et al., "The width of resection margins influences local recurrence in soft tissue sarcoma patients," European Journal of Surgical Oncology (EJSO), vol. 42, no. 6, pp. 899-906, 2016.

[4] M. Sluga, R. Windhager, S. Lang et al., "The role of surgery and resection margins in the treatment of Ewing???s sarcoma," Clinical Orthopaedics and Related Research, vol. 392, pp. 394-399, 2001.

[5] G. Bacci, A. Longhi, S. Mercuri, M. Versari, and F. Bertoni, "Prognostic factors in non-metastatic Ewing's sarcoma tumor of bone: an analysis of 579 patients treated at a single institution with adjuvant or neoadjuvant chemotherapy between 1972 and 1998," Acta Oncologica, vol. 45, no. 4, pp. 469-475, 2006.

[6] T. E. Bertrand, A. O. Cruz, D. Cheong, and G. D. Letson, "Do surgical margins affect local recurrence and survival in extremity, nonmetastatic, high-grade osteosarcoma?" Clinical Orthopaedics and Related Research, vol. 474, no. 3, pp. 677683, 2016.

[7] H. R. Dürr, Y. Bakhshai, H. Rechl, and P.-U. Tunn, “Tumorresektion: wie weit ist weit genug?" Der Unfallchirurg, vol. 117, no. 7, pp. 593-599, 2014.

[8] A. Gomez-Brouchet, E. Mascard, A. Siegfried et al., "Assessment of resection margins in bone sarcoma treated by neoadjuvant chemotherapy: literature review and guidelines of the bone group (GROUPOS) of the French sarcoma group and bone tumor study group (GSF-GETO/RESOS)," Orthopaedics \& Traumatology: Surgery \& Research, vol. 105, no. 4, pp. 773-780, 2019.

[9] R. D. Shelby, L. P. Suarez-Kelly, P. Y. Yu et al., "Neoadjuvant radiation improves margin-negative resection rates in extremity sarcoma but not survival," Journal of Surgical Oncology, vol. 121, no. 8, pp. 1249-1258, 2020.

[10] G. Bacci, A. Longhi, F. Bertoni, M. Versari, and P. Picci, "The role of surgical margins in treatment of Ewing's sarcoma family tumors: experience of a single institution with 512 patients treated with adjuvant and neoadjuvant chemotherapy," International Journal of Radiation Oncology*Biology*Physics, vol. 65, no. 3, pp. 766-772, 2006.

[11] S. Vandergugten, S. Y. Traore, O. Cartiaux, F. Lecouvet, C. Galant, and P.-L. Docquier, "MRI Evaluation of Resection Margins in Bone Tumour Surgery," Sarcoma, vol. 2014, Article ID 967848, 2014.

[12] L. Bellanova, T. Schubert, O. Cartiaux et al., "MRI-based assessment of safe margins in tumor surgery," Sarcoma, vol. 2014, Article ID 686790, 2014.

[13] L. Cannavò, D. Albano, C. Messina et al., "Accuracy of CT and MRI to assess resection margins in primary malignant bone tumours having histology as the reference standard," Clinical Radiology, vol. 74, no. 9, pp. 736.e13-736.e21, 2019.

[14] J. A. Abraham, B. Kenneally, K. Amer, and D. S. Geller, "Can navigation-assisted surgery help achieve negative margins in resection of pelvic and sacral tumors?" Clinical Orthopaedics and Related Research, vol. 476, no. 3, pp. 499-508, 2018.
[15] S. E. Bosma, A. H. G. Cleven, and P. D. S. Dijkstra, "Can navigation improve the ability to achieve tumor-free margins in pelvic and sacral primary bone sarcoma resections? A historically controlled study," Clinical Orthopaedics and Related Research, vol. 477, no. 7, pp. 1548-1559, 2019.

[16] M. J. Thompson and J. C. Shapton, S. E. Punt, C. N. Johnson, and E. U. Conrad, MRI identification of the osseous extent of pediatric bone sarcomas," Clinical Orthopaedics and Related Research, vol. 476, no. 3, pp. 559-564, 2018.

[17] C. Wittekind, C. C. Compton, F. L. Greene, and L. H. Sobin, "TNM residual tumor classification revisited," Cancer, vol. 94, no. 9, pp. 2511-2516, 2002.

[18] A. Rubin, H. Gannon, K. Inwards et al., "Protocol for the examination of specimens from patients with tumors of bone," Archives of Pathology \& Laboratory Medicine, vol. 134, no. 4, pp. e1-7, 2010.

[19] Cp-Bone-Primary-Tumors-2013-V3111.Pdf, https://documents. cap.org/protocols/cp-bone-primary-tumors-2013-v3111.pdf, 2013.

[20] WHO-OMS, WHO Classification of Tumours of Soft Tissue and Bone, WHO-OMS, Geneva, Switzerland, 2020, https://apps.who.int/bookorders/anglais/detart1.jsp? codlan $=1 \& \operatorname{cod} \operatorname{col}=70 \& \operatorname{cod} c \mathrm{ch}=4005 \#$.

[21] C. Thévenin-Lemoine, L. Destombes, J. Vial et al., "Planning for bone excision in ewing sarcoma," The Journal of Bone and Joint Surgery American, vol. 100, no. 1, pp. 13-20, 2018.

[22] P. Gulia, G. Subi, and Juvekar and Rekhi, "Comparison of MRI and Histopathology with Regard to Intramedullary Extent of Disease in Bone Sarcomas," Sarcoma, vol. 2019, 2019 Research Article, Article ID 7385470.

[23] S. Ahmad, J. Stevenson, and C. Mangham, G. Cribb and P. Cool, Accuracy of magnetic resonance imaging in planning the osseous resection margins of bony tumours in the proximal femur: based on coronal T1-weighted versus STIR images," Skeletal Radiology, vol. 43, no. 12, pp. 1679-1686, 2014.

[24] J. A. Cribb, Y. Wang, F. R. W. Van De Goot, and R. R. R. Gerretsen, "Statistical analysis of kerf mark measurements in bone," Forensic Science, Medicine, and Pathology, vol. 7, no. 1, pp. 53-62, 2011.

[25] J. Werier, X. Yao, J.-M. Caudrelier et al., "Evidence-based guideline recommendations on treatment strategies for localized Ewing's sarcoma of bone following neo-adjuvant chemotherapy," Surgical Oncology, vol. 25, no. 2, pp. 92-97, 2016. 